\title{
Olive oil phenols and their metabolites modulate nitric oxide balance in Human Aortic Endothelial Cells
}

Nitric oxide (NO) is an important vasodilator involved in the regulation of vascular homeostasis and plays a crucial role in maintaining a normal endothelial function ${ }^{(1)}$. NO modulates vasodilatation and blood flow regulation and regulates vessel homeostasis. Dietary polyphenols and their metabolites can improve vascular function ${ }^{(2)}$, through maintenance of local levels of NO. Thus, the molecular events involved are not fully understood ${ }^{(3)}$. The aim of this study was to investigate the effect of extra-virgin olive oil (EVOO) simple phenols tyrosol (Tyr) and hydroxytyrosol (HT), together with their methylated, glucuronide and sulfate metabolites, on NO balance at vascular level.

Primary Human Aortic Endothelial Cells (HAEC) were exposed to physiologically relevant concentrations $(1 \mu \mathrm{M})$ of the compounds or vehicle (methanol) for $24 \mathrm{~h}$ or $2 \mathrm{~h}$. Apocynin and $\mathrm{N \omega}$-Nitro-L-arginine (L-NNA) were used as additional controls. Nitric oxide levels, superoxide production, cyclic GMP (cGMP) levels, and Akt1 activation were determined. The statistical analysis of the data (at least 4 biological replicates) was conducted by one-way ANOVA followed by a Tukey post-hoc t-test using the Graphpad Prism 7 software.

Similarly to apocynin, all compounds tested showed the ability to enhance cellular NO levels, measured as DAF2-DA fluorescence intensity $(\mathrm{P}<0.01)$ and production of cGMP $(\mathrm{P}<0.05)$, and significantly decrease superoxide production $(\mathrm{P}<0.05)$. Protein expression results obtained through western blotting showed that some compounds were able to significantly increase Akt1 activation $(\mathrm{P}<$ 0.05), measured as the ratio of the phosphorylated (Ser 473) / total protein.

Overall, our results indicated that olive oil phenolics and their circulating metabolites can modulate NO balance, by decreasing its degradation (via reduced superoxide formation), and by enhancing its production through the Akt1/eNOS pathway.

\section{References}

1. Tousoulis D, Kampoli AM, Tentolouris C et al. (2012) Curr Vasc Pharmacol 1, 4-18.

2. Vauzour D, Rodriguez-Mateos A, Corona G et al. (2010) Nutrients 2, 1106-1131.

3. Corona G, Spencer JPE and Vauzour D. (2014) J Nutr Health Ageing 2, 125-132. 